\title{
APPLYING TRANSFORMATION CHARACTERISTICS To Solve The Multi Objective Linear Fractional Programming Problems
}

\begin{abstract}
Wen Pei
Department of Business Administration, Chung Hua University, Hsinchu, Taiwan, ROC

ABSTRACT

For some management programming problems, multiple objectives to be optimized rather than a single objective, and objectives can be expressed with ratio equations such as return/investment, operating profit/net-sales, profit/manufacturing cost, etc. In this paper, we proposed the transformation characteristics to solve the multi objective linear fractional programming (MOLFP) problems. If a MOLFP problem with both the numerators and the denominators of the objectives are linear functions and some technical linear restrictions are satisfied, then it is defined as a multi objective linear fractional programming problem MOLFPP in this research. The transformation characteristics are illustrated and the solution procedure and numerical example are presented.
\end{abstract}

\section{KEYWORDS}

Transformation Characteristics, MOLFP, MOLFPP

\section{INTRODUCTION}

Management programming problems are based upon estimated values. These problems have multiple objectives to be optimized rather than a single objective. Thus optimal solution to one objective function is not necessarily optimal for other objective functions and hence one need a solution as the compromise solution. In the meantime, for some management programming problems, objectives can be expressed in ratio equations such as return/investment, operating profit/net-sales, profit/manufacturing cost, etc. These multiple objective fractional programming models were first studied by Luhandjula [6]. Kornbluth and Steuer [4] have presented an algorithm for solving the MOLFP by combining aspects of multiple objective, single objective fractional programming and goal programming. Valipour et al. [9] suggested an iterative parametric approach for solving MOLFP problems which only uses linear programming to obtain efficient solutions and converges to a solution. Mishra et al. [7] presented a MOLFP approach for multi objective linear fuzzy goal programming problem. Li et al. [5] proposed a two-level linear fractional water management model based on interactive fuzzy programming. Saha et al. [8] proposed an approach for solving linear fractional programing problem by converting it into a single linear programming problem, which can be solved by using any type of linear fractional programming technique.

Zimmermann [10,11] first applied fuzzy set theory concept with choices of membership functions and derived a fuzzy linear program which is identical to the maximum program. $\mathrm{He}$ 
showed that solutions obtained by fuzzy linear programming are efficient solutions and also gives an optimal compromise solution. Luhandjula [6] solved MOLFP by applying fuzzy approach to overcome the computational difficulties of using conventional fractional programming approaches to solve multiple objective fractional programming problem. Charnes and Cooper [2] have shown that a linear fractional programming problem can be optimized by reducing it to two linear programs to solve MOLFP. Dutta et al [3] modified the linguistic approach of Luhandjula [6] by constructing the desirable membership functions. Chakraborty and Gupta [1] proposed a different methodology for solving MOLFP. The approach stated that suitable transformation should have been applied to formulate an equivalent multi objective linear programming and the resulting multi objective linear programming could be solved based on fuzzy set theoretic approach.

In this research, based on Chakraborty and Gupta [1], if a MOLFP problem with both the numerators and the denominators of the objectives are linear functions and some technical linear restrictions are satisfied, then it is defined as a MOLFPP. We propose the transformation characteristics to solve the MOLFPP. The transformation characteristics are illustrated and the solution procedure and numerical example presented.

\section{METHODS}

\subsection{The Transformation Characteristics of MOLFPP}

\subsubsection{Fuzzy Linear Programming}

Fuzzy linear programming is fuzzy set theory applied to linear multi criteria decision making problems. The multi objective linear fractional programming problem can be considered as a vector optimizing problem. The first step is to assign two values $U_{k}$ and $L_{k}$ as upper and lower bounds for each objective function $Z_{k}$ :

$U_{k}=$ Highest acceptable level of achievement for objective $k$

$L_{k}=$ Aspired level of achievement for objective $k$

Let

$d_{k}=U_{k}-L_{k}=$ the degradation allowance for objective $k$.

Takes an element $X$ that has a degree of membership in the $k$-th objective, denoted by a membership function $\mu_{k}(X)$, to transform the fuzzy model into a crisp single objective linear programming model of $\lambda$. The range of the membership function is $[0,1]$. 


$$
\mu_{k}(X)=\left\{\begin{array}{lll}
1 & \text { if } & Z_{k} \leq L_{k}, \\
1-\frac{Z_{k}-L_{k}}{U_{k}-L_{k}} & \text { if } & L_{k}<Z_{k}<U_{k}, \\
0 & \text { if } & Z_{k} \geq U_{k} .
\end{array}\right.
$$

This approach is similar, in many respects, to the weighted linear goal programming method.

\subsubsection{Linear Fractional Programming}

The general format of a classical linear fractional programming problem Charnes and Cooper [2] can be stated as

$\operatorname{Max} \frac{c^{T} x+\alpha}{d^{T} x+\beta}$

$$
\text { s.t. } x \in X=\left\{x \in R^{n} \mid A x\left(\begin{array}{l}
\leq \\
= \\
\geq
\end{array}\right) b, x \geq 0, b \in R^{m}\right\},
$$

where $c, d \in R^{n} ; \alpha, \beta \in R, X$ is nonempty and bounded.

We proposed the basic transformation characteristic of the original objective to solve the problem. The following transformation is proposed:

$$
\underset{x \in \Delta}{\operatorname{Max}} \frac{N(x)}{D(x)} \Leftrightarrow \operatorname{Min}_{x \in \Delta} \frac{D(x)}{N(x)} \Leftrightarrow \underset{x \in \Delta}{\operatorname{Min}} \frac{-D(x)}{-N(x)}
$$

\subsubsection{Multiple Objectives Linear Fractional Programming Problem}

The general format of maximizing MOLFPP can be written as

$$
\operatorname{Max} Z(x)=\left\{\begin{array}{c}
Z_{1}(x)=\frac{c_{1}^{T} x+\alpha_{i}}{d_{1}^{T} x+\beta_{i}} \\
Z_{2}(x)=\frac{c_{2}^{T} x+\alpha_{i}}{d_{2}^{T} x+\beta_{i}} \\
\cdot \\
\cdot \\
Z_{k}(x)=\frac{c_{k}^{T} x+\alpha_{i}}{d_{k}^{T} x+\beta_{i}}
\end{array}\right\}
$$


International Journal of Computer Science \& Information Technology (IJCSIT) Vol 9, No 2, April 2017

$$
\text { s.t. } x \in X=\left\{x \in R^{n} \mid A x\left(\begin{array}{l}
\leq \\
= \\
\geq
\end{array}\right) b, x \geq 0, b \in R^{m}\right\},
$$

Where $c_{i}, d_{i} \in R^{n} ; \alpha_{i}, \beta_{i} \in R, i=1,2, \ldots k, k \geq 2, X$ is nonempty and bounded.

Similarly, minimum problem can also be defined as

$\operatorname{Min} Z(x)=\left[Z_{1}(x), Z_{2}(x), \ldots, Z_{k}(x)\right]$

$$
\text { s.t. } x \in X=\left\{x \in R^{n} \mid A x\left(\begin{array}{l}
\leq \\
= \\
\geq
\end{array}\right) b, x \geq 0, b \in R^{m}\right\},
$$

Where $c_{i}, d_{i} \in R^{n} ; \alpha_{i}, \beta_{i} \in R, i=1,2, \ldots k, k \geq 2, X$ is nonempty and bounded,

with $Z_{i}(x)=\frac{c_{i}^{T} x+\alpha_{i}}{d_{i}^{T} x+\beta_{i}}=\frac{N_{i}(x)}{D_{i}(x)}$

The general format of minimum MOLFPP is as the following equivalent multi objective linear programming problem:

$\operatorname{Min} c_{i}^{T} y+\alpha_{i} t$,

s.t. $d_{i}^{T} y+\beta_{i} t=\gamma$

$A_{i} y-b_{i} t \leq 0$,

$y, t \geq 0, i=1,2, \ldots k, k \geq 2$.

The membership functions for $N_{i}(x)$ and $D_{i}(x)$ are as followed:

If $i \in I$, then

$\mu_{i}\left(t N_{i}(y / t)\right)$

$=\left\{\begin{array}{lll}0 & \text { if } & t N_{i}(y / t) \leq 0 \\ \frac{t N_{i}(y / t)-0}{\bar{Z}_{i}-0} & \text { if } & 0<t N_{i}(y / t)<\bar{Z}_{i} \\ 1 & \text { if } & t N_{i}(y / t) \geq \bar{Z}_{i}\end{array}\right.$

If $i \in I^{c}$, then

$\mu_{i}\left(t D_{i}(y / t)\right)$ 


$$
=\left\{\begin{array}{lll}
0 & \text { if } & t D_{i}(y / t) \leq 0 \\
\frac{t D_{i}(y / t)-0}{\bar{Z}_{i}-0} & \text { if } & 0<t D_{i}(y / t)<\bar{Z}_{i} \\
1 & \text { if } & t D_{i}(y / t) \geq \bar{Z}_{i}
\end{array}\right.
$$

The Zimmermann's $[10,11]$ operator is used to transform the equivalent multi objective linear programming problem into the crisp model as:

$\operatorname{Max} \lambda$

$$
\begin{aligned}
& \text { s.t. } \mu_{i}\left(t N_{i}(y / t)\right) \geq \lambda \text { for } i \in I, \\
& \mu_{i}\left(t D_{i}(y / t)\right) \geq \lambda \text { for } i \in I^{c}, \\
& t D_{i}(y / t) \leq 1 \quad \text { for } i \in I, \\
& -t N_{i}(y / t) \leq 1 \quad \text { for } i \in I^{c}, \\
& A(y / t)-b \leq 0, \\
& t>0, y \geq 0, \quad i=1,2, \ldots k, k \geq 2 .
\end{aligned}
$$

$I$ is a set such that $I=\left\{i: N_{i}(x) \geq 0\right.$ for some $\left.x \in \Delta\right\}$ and $I^{c}=\left\{i: N_{i}(x)<0\right.$ for each $\left.x \in \Delta\right\}$ where $I \cup I^{c}=\{1,2, \ldots, k\}$. The computing of $\bar{Z}_{i}$, is proceeded as "if $i \in I$, then it may assume the maximum aspiration level is $\bar{Z}_{i}=Z_{i}^{*}$, and if $i \in I^{c}$, then $\bar{Z}_{i}=-1 / Z_{i}^{*}$." The method proposed in this paper suggests that with $t=0$, by Charnes and Cooper [2] method, the problem could not be solved, the transformation characteristics can be used to solve the MOLFPP.

\section{RESULTS AND DISCUSSION}

The solution procedure is stated and numerical examples adopted from Chakraborty and Gupta [1] are used to show the transformation characteristics.

\subsection{Solution Procedure}

The transformation characteristics are used to solve MOLFPP when $t=0$ from the original problems. The following procedure is developed:

Step 1. Solve the original MOLFPP by Charnes and Cooper [2] .

Step 2. If $t=0$, the proposed methodology is applied.

Step 3. Solve the problem by Zimmermann's [10,11] operator to transform the equivalent multi objective linear programming problem into the crisp model.

\subsection{Numerical Examples}

Let's consider a MOLFP with two objectives as follows: 
$\operatorname{Max} Z(x)=\left(\begin{array}{l}Z_{1}(x)=\frac{-3 x_{1}+2 x_{2}}{x_{1}+x_{2}+3}, \\ Z_{2}(x)=\frac{7 x_{1}+x_{2}}{5 x_{1}+2 x_{2}+1}\end{array}\right)$

s.t. $x_{1}-x_{2} \geq 1$,

$2 x_{1}+3 x_{2} \leq 15$,

$x_{1} \geq 3$,

$x_{i} \geq 0, i=1,2$.

Solve the MOLFP by Charnes and Cooper [2] approach.

$\operatorname{Max}\left(\begin{array}{l}\left.f_{1}(y, t)=-3 y_{1}+2 y_{2}\right) \\ \left.f_{2}(y, t)=7 y_{1}+y_{2}\right)\end{array}\right)$

s.t. $y_{1}+y_{2}+3 t \leq 1$,

$5 y_{1}+2 y_{2}+t \leq 1$,

$y_{1}-y_{2}-t \geq 0$,

$2 y_{1}-3 y_{2}-15 t \leq 0$,

$y_{1}-3 t \geq 0$,

$y_{i}, t \geq 0, i=1,2$.

Where $y_{1}=0, y_{2}=0, t=0$ for $f_{1}(y, t)$, and $y_{1}=0.194805, y_{2}=0, t=0.025974$

for $f_{2}(y, t)$. Thus $\left(U_{1}, L_{1}\right)=(0,-0.584415),\left(U_{2}, L_{2}\right)=(1.3636,0)$

With Zimmerman's [10,11] approach, the above multi objective linear programming problem could be solved. The solution of the problem is obtained as $\lambda=0.7741981, y_{1}=0.131962$, $y_{2}=0.131962$, and $t=0$. The original problem could be translated into the following MOLFPP:

$\operatorname{Min} Z(x)=\left(\begin{array}{l}Z_{1}(x)=\frac{x_{1}+x_{2}+3}{-3 x_{1}+2 x_{2}}, \\ Z_{2}(x)=\frac{5 x_{1}+2 x_{2}+1}{7 x_{1}+x_{2}}\end{array}\right)$

s.t. $x_{1}-x_{2} \geq 1$,

$2 x_{1}+3 x_{2} \leq 15$,

$x_{1} \geq 3$,

$x_{i} \geq 0, i=1,2$. 
The equivalent MOLFPP is as followed:

$\operatorname{Min}\left(\begin{array}{l}f_{1}(y, t)=-y_{1}-y_{2}-3 t \\ f_{2}(y, t)=5 y_{1}+2 y_{2}+t\end{array}\right)$

s.t. $3 y_{1}-2 y_{2} \leq 1$,

$7 y_{1}+y_{2} \leq 1$

$y_{1}-y_{2}-t \geq 0$,

$2 y_{1}+3 y_{2}-15 t \leq 0$

$y_{1}-3 t \geq 0$,

$y_{i}, i=1,2, t \geq 0$.

The solution are $\left(U_{1}, L_{1}\right)=(0,-0.3478), \quad\left(U_{2}, L_{2}\right)=(0.8696,0), \quad \lambda=0.49998$, $y_{1}=0.065215, y_{2}=0.043477$, and $t=0.021738$. The solution of the original problem is:

$x_{1}=3, x_{2}=2, Z_{1}=\frac{-5}{8}, Z_{2}=\frac{23}{20}$.

\section{Conclusions}

The transformation characteristics to solve MOLFPP based on fuzzy set theoretic approach are proposed in this research. The MOLFPP can be transformed into the equivalent appropriate multi objective linear programming problem by using the transformation characteristics. The resulting multi objective linear programming problem is solved using fuzzy set theoretic approach by membership functions. Numerical example is utilized to illustrate the proposed methodology.

\section{REFERENCE}

[1] Chakraborty, M. and Gupta, S. (2002), "Fuzzy mathematical programming for multi objective linear fractional programming problem," Fuzzy Sets and Systems, Vol. 125, pp. 335-342.

[2] Charnes, A. and Cooper, W.W. (1962), "Programming with linear fractionals," Naval Research Logistics Quarterly, Vol. 9, pp. 181-186.

[3] Dutta, D., Tiwari, R.N. and Rao, J.R. (1992), "Multiple objective linear fractional programming - A fuzzy set theoretic approach," Fuzzy Sets and Systems, Vol. 52, pp. 39-45.

[4] Kornbluth, J.S.H. and Steuer, R.E. (1981), "Multiple objective linear fractional programming," Management Science, Vol. 27, pp. 1024-1039.

[5] Li, M., Guo, P., \& Ren, C. (2015), "Water resources management models based on two-level linear fractional programming method under uncertainty," Journal of Water Resources Planning and Management, 141(9), 05015001.

[6] Luhandjula, M.K. (1984), "Fuzzy approaches for multiple objective linear fractional optimization," Fuzzy Sets and Systems, Vol. 13, pp. 11-23.

[7] Mishra, B., Nishad, A. K., \& Singh, S. R. (2014), "Fuzzy Multi-fractional Programming for Land Use Planning in Agricultural Production System, ” Fuzzy Information and Engineering, 6(2), 245-262. 
International Journal of Computer Science \& Information Technology (IJCSIT) Vol 9, No 2, April 2017

[8] Saha, S. K., Hossain, M. R., Uddin, M. K., \& Mondal, R. N. (2015), “ A New Approach of Solving Linear Fractional Programming Problem (LFP) by Using Computer Algorithm," Open Journal of Optimization, 4(03), 74.

[9] Valipour, E., Yaghoobi, M. A., \& Mashinchi, M. (2014), "An iterative approach to solve multi objective linear fractional programming problems," Applied Mathematical Modelling, 38(1), 38-49.

[10] Zimmermann, H.J. (1976), "Description and optimization of fuzzy systems," International Journal of General Systems, Vol. 2, pp. 209-215.

[11] Zimmermann, H.J. (1978), "Fuzzy programming and linear programming with several objective functions," Fuzzy Sets and Systems, Vol. 1, pp. 45-55. 